\title{
13. RADIOLARIANS FROM THE SEA OF JAPAN: LEG 128 ${ }^{1}$
}

\author{
Hsin-Yi Ling ${ }^{2}$
}

\begin{abstract}
The analysis of radiolarians from Japan Sea subsurface sediments recovered during Leg 128 of the Ocean Drilling Program reveals that a warm-water assemblage similar to that of the North Pacific was replaced by unique post-middle Miocene faunas probably as a result of the restriction of oceanographic circulation. The modern fauna was gradually established only in the Pleistocene. No attempt was made to establish the radiolarian zonation because of low species diversity and the absence of generally recognized index forms in the North Pacific. In the diagenetically altered quartz section, however, a radiolarian assemblage correlative to the middle Miocene Cyrtocapsella tetrapera Zone of western Honshu was identified from Hole 799B.
\end{abstract}

\section{INTRODUCTION}

The results of the analysis of Radiolaria from subsurface sediments recovered during Leg 31 of the Deep Sea Drilling Project (DSDP) suggest that a major radiolarian faunal change took place after the late early to early middle Miocene in the Japan Sea and that unique, colder post-middle Miocene radiolarian faunas replaced the warm open-ocean assemblage. Consequently, the radiolarian zonation established in the open North Pacific was not applicable to the area (Ling, 1975). Leg 128 of the Ocean Drilling Program (ODP) in 1989 provided a second opportunity to examine these unique assemblages, identify the datum levels, and calibrate them with shipboard magnetostratigraphy. Unexpectedly, the rather continuous submarine sections also provide an opportunity to observe radiolarian preservation in the downcore silica transformation from opal-A to opal-CT, and opal-CT to quartz stages.

The subsurface sediments were recovered from Sites $798\left(37^{\circ}\right.$ $38.32^{\prime} \mathrm{N}, 134^{\circ} 79.976^{\prime} \mathrm{E}, 903.1 \mathrm{~m}$ water depth $)$ and $799\left(39^{\circ} 22.046^{\prime} \mathrm{N}\right.$, $133^{\circ} 86.685^{\prime} \mathrm{E}, 2073.0 \mathrm{~m}$ water depth) (Fig. 1). Sample preparation methods and the location descriptions of the illustrated specimens are previously described (see Ling, 1973).

All microslides used for the present investigation, including the figured specimens, will be permanently deposited in the Micropaleontology Collection of the Department of Geology at Northern Illinois University.

\section{SITE SUMMARIES}

\section{Site 798}

Radiolarians are generally moderately well preserved although in low abundance in most of the samples from Site 798 (Tables 1-3). The absence of age indicators widely recognized in the North Pacific late Neogene sequences precludes any attempt at zonation.

In Hole 798A (Table 1), the uppermost submarine section, from the subsurface down to Sample 128-798A-1H-3, 29-31 cm (0-3.29 $\mathrm{mbsf}$ ), the radiolarians are typical of middle- to high-latitude assemblages and consist of Cycladophora davisiana, Stylochlamidium venustum, and Nephrospyris? pervia (two types). From Sample 128$798 \mathrm{~A}-1 \mathrm{H}-5,30-32 \mathrm{~cm}$, to at least Sample 128-798A-10H-1, 31-33 $\mathrm{cm},(6.30-84.71 \mathrm{mbsf})$, the rare and spotty occurrences of various modern warm to temperate forms were noted, including Amphirhopalum ypsilon, Didymocyrtis tetrathalamus tetrathalamus, Euchitonia elegans, E. furcata, Spongaster tetras tetras, and Tetrapyle

\footnotetext{
' Pisciotto, K. A., Ingle, J. C., Jr., von Breymann, M. T., Barron, J., et al., 1992. Proc. ODP, Sci. Results, 127/128, Pt. 1: College Station, TX (Ocean Drilling Program).

${ }^{2}$ Department of Geology, Northern Illinois University, DeKalb, IL 60115, U.S.A.
}

octacantha. This Quaternary warm interval can be traced along the eastern Sea of Japan, because among the four sites drilled during DSDP Leg 31 cruise, only Site 299 from the northeast Yamato Basin (see Fig. 1) yielded similar warm-water species, including Amphirhopalum upsilon and Spongaster tetras tetras (Ling, 1975). At the same time, it may also suggest the geographical limitations of the Tsushima Current (warm) in the area (Fig. 2). Future analysis of the samples drilled at Sites 794, 795, and 796 during Leg 127 should answer this specific question. In the bottom section of the hole, 84.71-143.3 mbsf (Core 128-798A-17X), radiolarians are rare to few in abundance and without any age-diagnostic species, except for the first appearance datum (FAD) of both Cycladophora davisiana davisiana and $C$. d. cornutoides in Sample 128-798A-13H-3, 31-33 cm (126.71 mbsf), and the last appearance datum (LAD) of Druppatractus acquilonius in Sample 128-798A-15H-7, 43-46 cm (142.13 mbsf).

Sample analysis in Hole 798B (Table 2) began with Section 128798B-14-CC (132.9 mbsf), but radiolarians were either too rare or the species encountered were non-age-diagnostic. The low frequency but continuous presence of Anthocorys(?) akitaensis from Sample 128798B-41X-7, 40-43 cm (392.3 mbsf) and Stichocorys delmontensis together with Thecosphera japonica down to Section 128-798B-47XCC (450.4 mbsf), the last radiolarian-bearing sediments from this site, is still within the Pliocene. Starting from Section 128-798B-48X-CC (460.1 mbsf) to the bottom of the hole, Section 128-798B-54X-CC (517.9 mbsf), radiolarians were completely absent.

Taking an opportunity for continuous APC coring for heat flow at Hole $798 \mathrm{C}$, only core-catcher samples, $128-798 \mathrm{C}-1 \mathrm{H}-\mathrm{CC}$ through $128-798 \mathrm{C}-13 \mathrm{H}-\mathrm{CC}$ (120.1 mbsf) were analyzed to verify the faunal composition observed from Hole 798A (Table 3).

\section{Site 799}

As at Site 798A, low species abundance and the absence of index forms make any attempt at zonation difficult (Table 4). The occurrence of Cycladophora davisiana davisiana continues intermittently from the uppermost Sample 128-799A-1H-CC (1.2 mbsf) down to Sample 128-799A-16H-6. 80-82 cm (144.1 mbsf). In Section 128799A-13H CC (126.5 mbsf), the rare occurrence of Sphaeropyle langii was observed to continue sporadically to Section 128-799A16H-CC (145.5 mbsf). In Samples 128-799A-18H-CC (164.8 mbsf) through $128-799 \mathrm{~A}-44 \mathrm{X}-\mathrm{CC}$ (403.9 mbsf), radiolarians are too rare in occurrence to make any age assignments possible.

In Sample 128-799A-45X-2, 73-75 cm (406.13 mbsf), the wellpreserved specimens of Lychnocanoma sp., and Anthocorys? akitaensis were observed, and in Sample 128-799A-46X-6, 80-82 cm (412.2 mbsf), a few specimens of Stichocorys delmontensis were recognized. The occurrence of Lychnocanoma sp. continues to Section 129-799A49X-CC (452.3 mbsf), whereas Anthocorys? akitaensis and Sticho- 


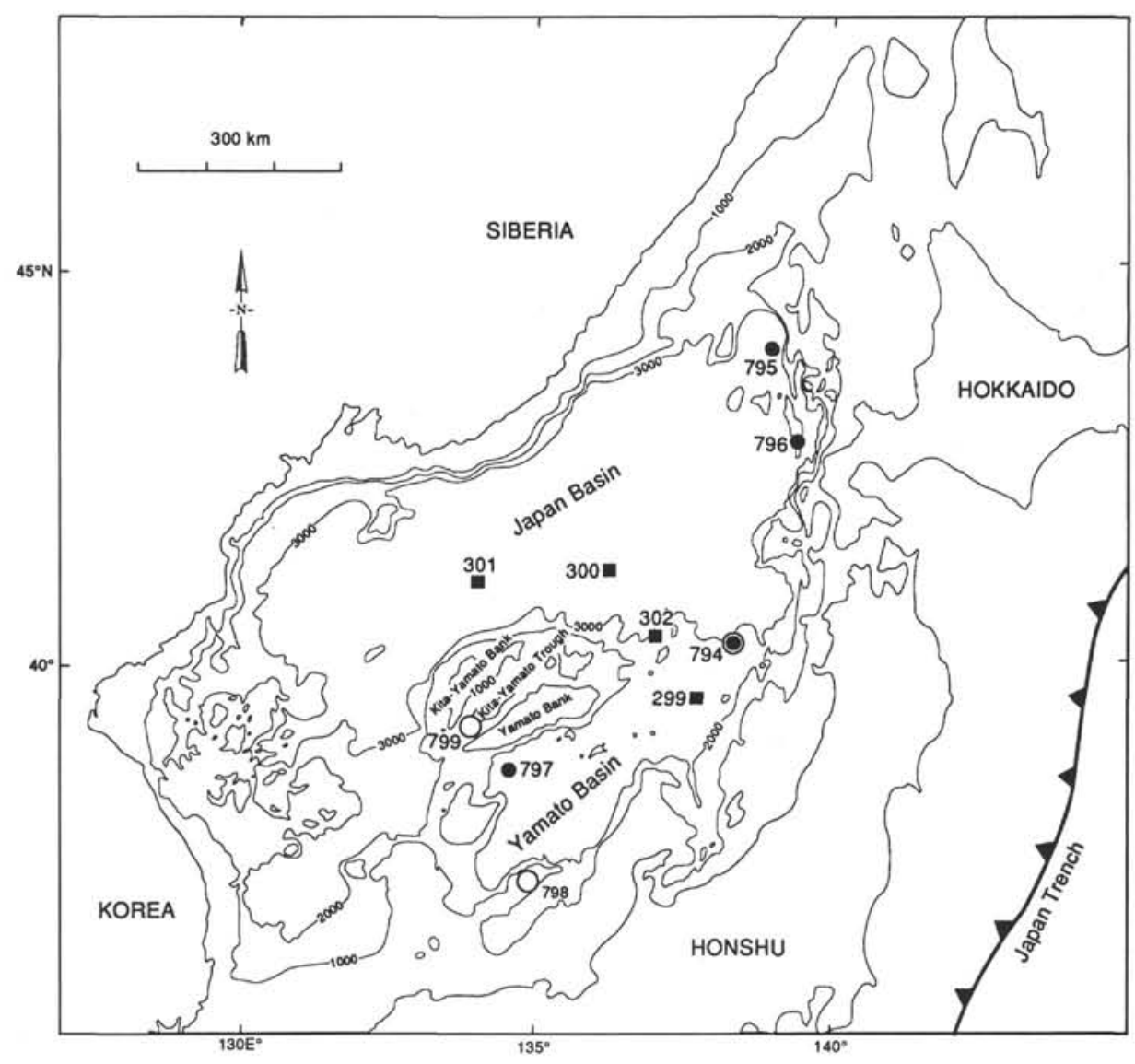

Figure 1. Index map showing the location of ODP Leg 128 sites (open circles) in relation to the sites drilled previously during ODP Leg 127 (solid circles), and DSDP Leg 31 (solid squares). Site 794 was drilled during both Legs 127 and 128, but was not cored during the latter. Bathymetry in meters.

corys delmontensis were recovered again in Section 128-799A-51XCC (466.9 mbsf).

Finding these species below Core 128-799A-45X is significant for the following reasons:

First, although the geological occurrence of these taxa in the Sea of Japan coastal region of Honshu extends upward to the Thecosphaera japonica Zone (late late Miocene to early early Pliocene), they are generally observed in the Lychnocanoma nipponica (= Lychnocanium nipponicum) Zone (late middle Miocene to early late Miocene) of Nakaseko and Sugano (1973).

Their occurrence in Hole 799A is thus compatible with the interpretations from shipboard paleomagnetic analysis.

Second, a similar assemblage was reported from Site 302 (Ling, 1975), the only known such occurrence from submarine sediments of the Sea of Japan, and its age is considered within the Denticula (= Neodenticula) kamtchatica (diatom) Zone of Koizumi (1975), and Koizumi and Tanimura (1985). Thus, these two occurrences can be considered time-correlative.

Third, their occurrence suggests that some of the radiolarian species can survive the critical silica diagenetic transformation from opal-A to opal-CT. According to the shipboard chemical analysis (Ingle, Suyehiro, von Breymann, et al., 1990), the opal-A/opal-CT boundary for the Hole 799A sediments is between Sections 128-799A-46X-CC and 128-799A48X-CC (423.3-432.9 mbsf). The limited occurrence of Lychnocanoma sp. together with Stichocorys delmontensis in this section suggests that the samples from Sections 128-799A-46X-CC (494.6 mbsf) to 128$799 \mathrm{~A}-51 \mathrm{X}-\mathrm{CC}$ (466.9 mbsf) can be assigned to the late Miocene. The deepest core of the hole, 128-799A-52X (466.9-468.7 mbsf), was barren of radiolarians.

Drilling of Hole 799B started from the opal-CT zone (Table 5), therefore, the absence and/or poor preservation of radiolarians, if preserved, was anticipated. Single specimens of Spongodiscus in Section 128-799B-5R-CC (494.6 mbsf) and 128-799B-7R-CC (509.3 mbsf), and Porodiscus sp. in Sections 128-799B-9R-CC (528.6 mbsf) and 128-799B10R-CC ( $538.3 \mathrm{mbsf}$ ) were observed, but all specimens had been totally diagenetically altered so that in most cases their identification even at a generic level was difficult. In Sections 128-799B-31R-CC (740.5 mbsf), 128-799B-32R-CC (750.2 mbsf), and 128-799B-38-CC (808.1 mbsf), in addition to Cyrtocapsella tetrapera and Spongodiscus spp., spherical forms probably belonging to the genus Thecosphaera were observed, and Section 128-799B-48R-CC (904.2 mbsf) yielded moderately well-preserved, diagenetically altered specimens of Cyrtocapsella tetrapera, Didymocyrtis mammifera, and Lithopera renzae renzae. Therefore, at least the section from 740.5 to $904.2 \mathrm{mbsf}$ of Hole 799B can be assigned to the Cyrtocapsella tetrapera Zone of Nakaseko and Sugano (1973) (not Riedel and Sanfilippo, 1978) from the coastal region of Honshu, which is regarded as either late early Miocene or early middle Miocene (Berggren et al., 1985; Sanfilippo et al., 1985). These forms have been reported only from the middle- to low-latitude regions, suggesting the existence of warm-water conditions during deposition.

Except for Section 128-799B-50R-CC (923.3 mbsf), which contains specimens of Spongodiscus, radiolarians were completely absent below Section 128-799B-49R-CC (913.6 mbsf) to the bottom of the hole (Section 128-799B-67R-CC; $1084.0 \mathrm{mbsf}$ ). 
Table 1. Distribution of radiolarians, Hole 798A.

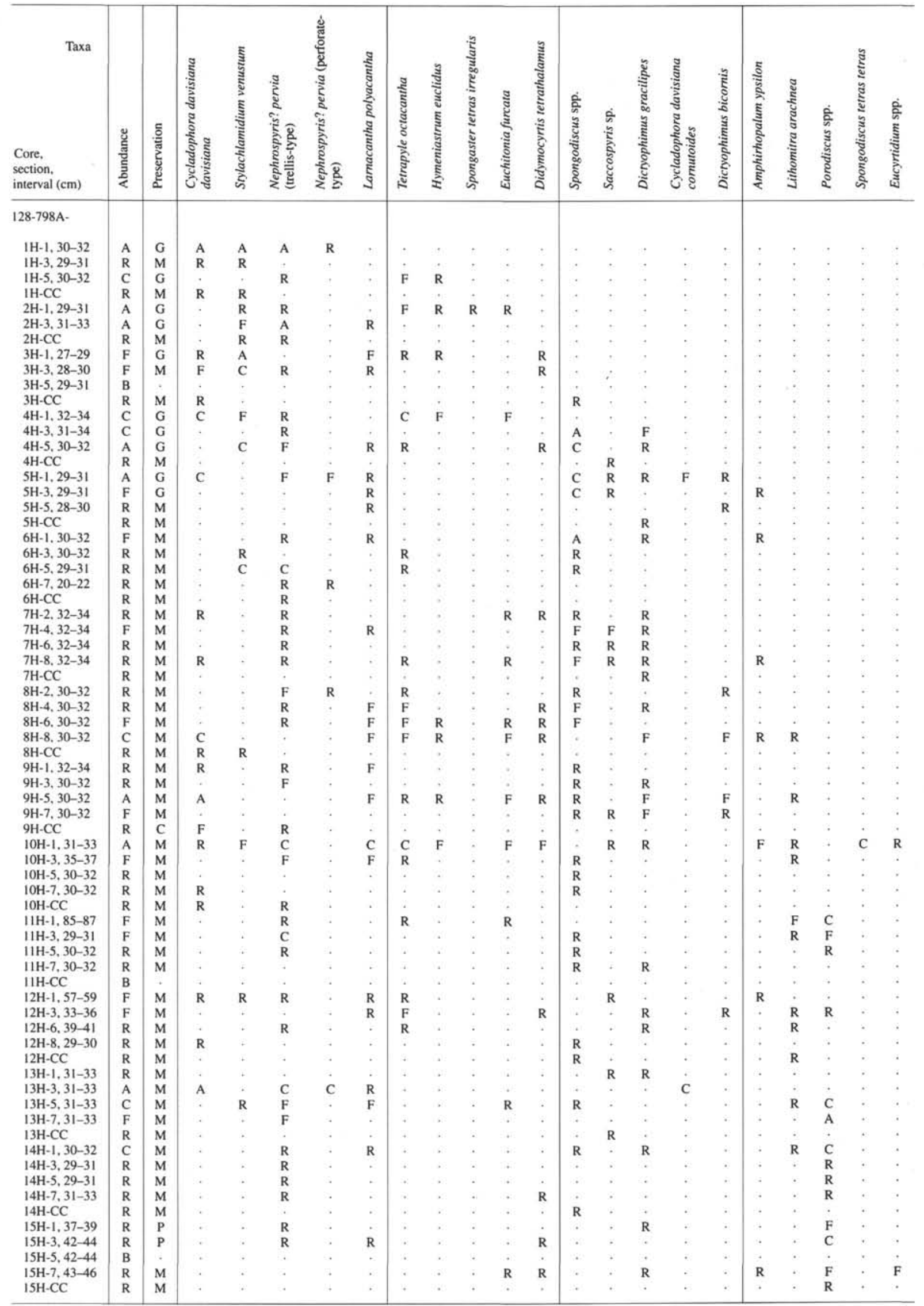




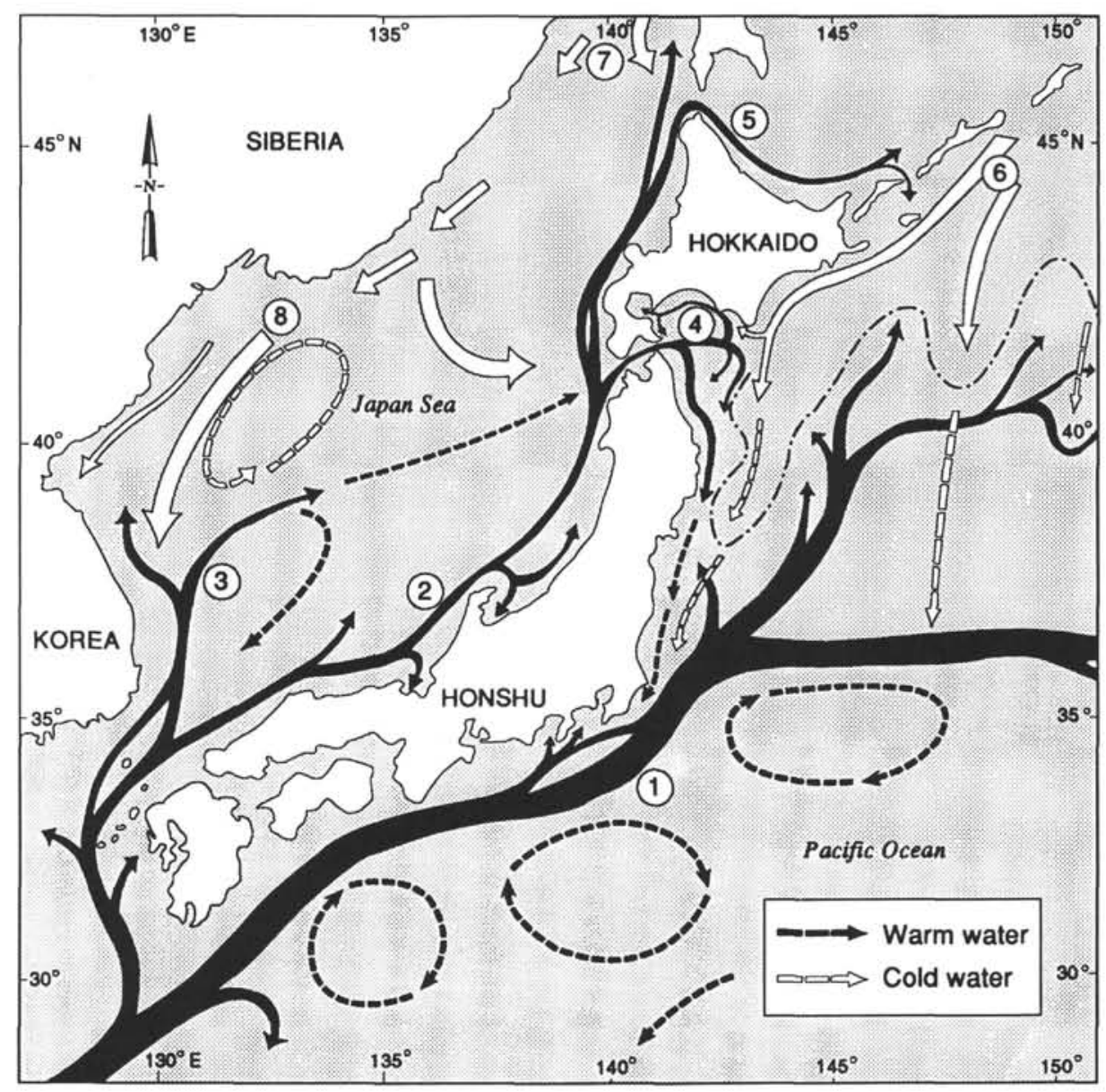

Figure 2. Current systems in the upper layer of the Japan Sea and adjacent waters (after Kawai, 1972). 1, Kuroshio; 2, Tsushima Current (warm); 3, East Korea Current (warm); 4, Soya Current (warm); 5, Tsugaru Current (warm); 6, Oyashio; 7, Liman Current; 8, North Korea Current.

\section{REMARKS ON POTENTIAL RADIOLARIAN DATUM LEVELS}

As reported earlier, aside from recognizing the wisely known Cyrtocapsella tetrapera Zone (Nakaseko and Sugano, 1973) of the region for the interval from Sections 128-799B-31R-CC through 128-799B-48R-CC (740.5-904.2 mbsf), no other radiolarian zones of the North Pacific or Japanese land sections could be identified or established from the subsurface sediments at Sites 798 and 799.

However, some of the radiolarian events observed during the study may have future biostratigraphic potential. They are discussed, in descending order, as follows:

1. FAD of Cycladophora davisiana davisiana: The initial appearance of $C$. d. davisiana has been suggested by Alexandrovich and Hays (in Tamaki, Pisciotto, Allan, et al., 1990) at 2.5-2.6 Ma. This event was observed at a much younger age at Leg 128: between Samples 128-798A-13H-3, 31-33 cm, and 128-798A-13H-5, 31-33 $\mathrm{cm}$, (126.71-129.71 mbsf) slightly above the LAD of Helicosphaera sellii (calcareous nannofossil) at $1.19 \mathrm{Ma}$ and $129.91 \mathrm{mbsf}$ (Ingle, Suyehiro, von Breymann, et al., 1990); between Sample 128-799A$16 \mathrm{H}-6,80-82 \mathrm{~cm}$, and 128-799A-16H-CC (144.10-145.5 mbsf), which is below the $\mathrm{LAD}$ of Ammodochium rectangulare (ebridian) that located Sample 128-799A-15H-4, 76-78 cm, and 128-799A$15 \mathrm{H}-5.76-78 \mathrm{~cm}(131.36-132.86 \mathrm{mbsf}$; Ling, this volume) and is at approximately $1.8 \mathrm{Ma}$. Thus, in spite of slight age differences, it seems that this datum is either early Pleistocene or latest Pliocene for the Leg 128 sediments.

2. LAD of Druppatractus acquilonius: This datum was recognized in the North Pacific just above the LAD of Stylatractus universus at $0.4 \mathrm{Ma}$ (Hays, 1970) or 0.41 Ma (Hays and Shackleton, 1976). In the Leg 128 samples, the LAD of this species was observed between Samples 128-798 A-15H-7, 43-46 cm, and 128-798A-15HCC (142.13-142.5 mbsf) and between Samples 128-799A-15H-5, 76-78 cm, and 128-799A-15H-6, 76-78 cm, (132.86-134.36 cm) which is definitely older than $1 \mathrm{Ma}$. Further confirmation of this level from the Japan Sea sediments presents the interesting question of why the species became extinct much earlier in the Sea of Japan than in the North Pacific.

3. LAD of Anthocorys(?) akitaensis: A sharp decline in the abundance of this species as well as that of Lychnocanoma nipponica and Theocyrtis redondoensis has been widely recognized in the land sections of the western side of Honshu as defining the top of the Lychnocanoma nipponica Zone (Nakaseko and Sugano, 1973).

This datum can be recognized by the FAD of Thecosphaera japonica and evolutionary transition of Stichocorys peregrina from its ancestor, S. delmontensis (Takayanagi et al., 1979), and the latter is dated 6.3 Ma in the equatorial Pacific sediments (Theyer et al., 1978).

However, in the Hole 798B and 799A sediments, the LAD of Anthocorys? akitaensis (Sample 128-798B-41X-7, 40-43 cm; 392.30 mbsf) (and Lychonocanoma sp. in Sample 128-799A-45X-2, 73-75 $\mathrm{cm} ; 406.13 \mathrm{mbsf}$ ) is younger than the LAD of Stichocorys delmon- 
Table 2. Distribution of radiolarians, Hole 798B.

\begin{tabular}{|c|c|c|c|c|c|c|c|c|c|c|c|c|c|c|c|c|c|c|}
\hline $\begin{array}{l}\text { Core, section, } \\
\text { interval }(\mathrm{cm})\end{array}$ & 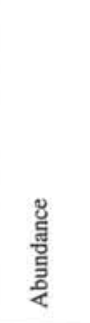 & 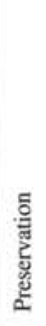 & 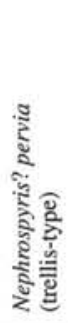 & 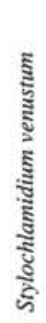 & 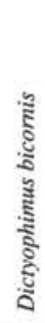 & 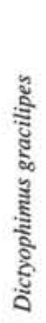 & 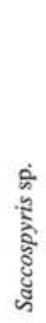 & 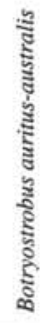 & 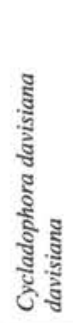 & 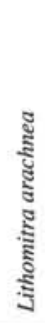 & 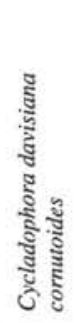 & 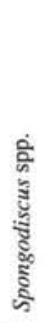 & 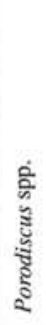 & 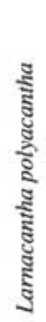 & 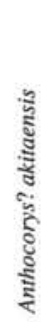 & 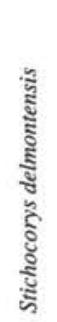 & 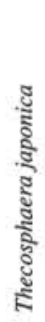 & 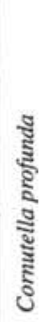 \\
\hline \multicolumn{19}{|l|}{$128-798 \mathrm{~B}-$} \\
\hline $14 \mathrm{H}-\mathrm{CC}$ & $R$ & M & $\mathrm{R}$ & $\mathrm{R}$ & & . & & . & . & . & . & & . & & . & . & . & . \\
\hline $15 \mathrm{H}-\mathrm{CC}$ & $\mathrm{R}$ & M & & . & $\mathbf{R}$ & $\mathrm{R}$ & $\mathbf{R}$ & & . & . & . & . & . & . & . & . & . & . \\
\hline $16 \mathrm{X}-\mathrm{CC}$ & $\mathrm{R}$ & M & . & . & $\mathrm{R}$ & . & . & $\mathrm{R}$ & . & . & . & . & . & . & . & . & . & . \\
\hline $17 \mathrm{X}-\mathrm{CC}$ & $\mathbf{R}$ & M & $\mathbf{R}$ & $\mathrm{R}$ & . & & . & & . & . & . & . & . & . & . & . & . & . \\
\hline $18 \mathrm{X}-\mathrm{CC}$ & $\mathrm{R}$ & M & . & & . & & . & & $\mathbf{R}$ & . & . & . & . & . & . & . & . & . \\
\hline $19 \mathrm{X}-\mathrm{CC}$ & B & . & . & . & . & & . & & . & . & . & . & . & & . & & . & . \\
\hline 20X-CC & $\mathrm{R}$ & M & . & & . & & . & & $\mathbf{R}$ & . & . & . & . & . & . & & , & . \\
\hline $21 \mathrm{X}-\mathrm{CC}$ & $\mathrm{R}$ & M & $\mathbf{R}$ & & . & & . & . & & & . & . & . & & . & & . & . \\
\hline $22 \mathrm{X}-\mathrm{CC}$ & B & & & . & . & . & . & . & . & . & & . & . & + & . & . & & . \\
\hline $23 \mathrm{X}-\mathrm{CC}$ & $\mathrm{R}$ & M & . & . & . & . & . & . & . & $\mathrm{R}$ & . & . & . & . & . & . & & . \\
\hline $24 \mathrm{X}-\mathrm{CC}$ & R & M & & . & . & . & . & . & . & $\mathrm{R}$ & . & . & . & . & . & & & . \\
\hline $25 \mathrm{X}-\mathrm{CC}$ & $\mathrm{R}$ & M & . & . & . & . & $\mathrm{R}$ & . & . & R & & . & , & . & . & . & . & . \\
\hline $26 \mathrm{X}-\mathrm{CC}$ & $\mathrm{R}$ & M & & . & . & . & . & . & . & $\mathrm{R}$ & . & . & . & . & . & . & & . \\
\hline $27 \mathrm{X}-\mathrm{CC}$ & $\mathrm{R}$ & M & . & . & . & . & $\mathrm{R}$ & . & . & $\mathrm{R}$ & . & . & . & . & . & . & & . \\
\hline $28 \mathrm{X}-\mathrm{CC}$ & R & M & & $\mathbf{R}$ & $\mathbf{R}$ & $\mathrm{R}$ & . & . & . & $\because$ & . & . & . & . & . & . & & . \\
\hline $30 \mathrm{X}-\mathrm{CC}$ & R & M & & . & . & ?. &. & . & . & . & . & $\mathrm{R}$ & & . & . & . & & . \\
\hline $31 \mathrm{X}-\mathrm{CC}$ & $\mathrm{R}$ & M & & . & . & . & . & . & . & $\mathrm{R}$ & . & . & . & . & . & . & . & . \\
\hline $32 \mathrm{X}-\mathrm{CC}$ & $\mathrm{R}$ & M & . & . & . & . & . & . & . & & $\mathrm{R}$ & . & & & . & . & . & . \\
\hline $33 \mathrm{X}-\mathrm{CC}$ & R & M & . & . & . & . & . & $\mathrm{R}$ & . & & ? & . & & . & . & . & . & . \\
\hline $34 \mathrm{X}-\mathrm{CC}$ & B & . & . & . & . & . & . & . & . & & & . & & . & & & . & \\
\hline $35 \mathrm{X}-\mathrm{CC}$ & R & M & . & . & . & . & . & . & . & $\mathrm{R}$ & . & . & . & . & & & & . \\
\hline $36 \mathrm{X}-\mathrm{CC}$ & R & M & $\mathbf{R}$ & . & . & . & & . & . & R & . & . & . & . & & . & . & \\
\hline $37 \mathrm{X}-\mathrm{CC}$ & B & & . & . & . & . & . & . & & ? & & . & . & . & . & & . & \\
\hline $38 \mathrm{X}-\mathrm{CC}$ & $\mathrm{R}$ & M & & . & . & . & $\mathrm{R}$ & . & . & & . & . & . & . & . & . & . & \\
\hline $39 \mathrm{X}-\mathrm{CC}$ & $\mathrm{R}$ & M & . & . & . & . & $\mathrm{R}$ & . & . & . & & & . & . & . & . & & . \\
\hline $40 \mathrm{X}-\mathrm{CC}$ & $\mathrm{R}$ & M & . & . & . & . & : & . & . & $\mathbf{R}$ & & & . & . & . & & . & \\
\hline $41 \times-5,57-59$ & B & & . & . & . & . & . & . & . & . & . & & . & . & . & . & . & \\
\hline $41 X-7,40-43$ & $\mathrm{~F}$ & M & $\mathrm{R}$ & . & . & . & $\mathbf{R}$ & . & . & $\mathbf{R}$ & $\mathrm{R}$ & $\mathrm{R}$ & $\mathrm{R}$ & $\mathrm{R}$ & $\mathbf{R}$ & . & & . \\
\hline $41 \mathrm{X}-\mathrm{CC}$ & B & . & . & . & . & . & : & . & . & . & . & . & . & . & ? & & . & .. \\
\hline $42 X-1,25-27$ & $\mathrm{R}$ & M & . & & . & & . & & . & $\mathrm{R}$ & . & $\mathrm{R}$ & . & $\mathbf{R}$ & . & . & . & . \\
\hline $42 X-3,25-27$ & $\mathrm{R}$ & M & $\mathbf{R}$ & . & . & & $\mathbf{R}$ & & . & . & & & . & . & $\mathbf{R}$ & . & . & . \\
\hline $42 X-5,45-47$ & $\mathrm{R}$ & M & . & . & . & & . & & . & $\mathrm{R}$ & & & . & $\mathbf{R}$ & $\mathrm{R}$ & . & . & . \\
\hline $42 \times-7,45-47$ & B & . & . & . & . & . & . & . & . & & . & & . & . & . & . & . & . \\
\hline $42 \mathrm{X}-\mathrm{CC}$ & $\mathrm{R}$ & M & . & . & . & . & . & . & . & . & . & $\mathrm{R}$ & . & . & . & . & . & . \\
\hline $43 \mathrm{X}-1,30-32$ & $\mathrm{R}$ & M & . & . & . & . & . & . & . & $\mathrm{R}$ & . & . & . & $\therefore$ & $\mathbf{R}$ & , & . & . \\
\hline $43 X-3,31-33$ & B & . & . & . & . & & . & . & . & & & . & . & . & & . & . & . \\
\hline $43 \times-5,32-34$ & $\mathrm{R}$ & M & . & & . & & . & . & . & & . & . & . & . & $\mathrm{R}$ & . & . & . \\
\hline $43 \mathrm{X}-\mathrm{CC}$ & $\mathrm{R}$ & M & . & & & . & . & . & . & $\mathrm{R}$ & . & . & . & . & & . & . & . \\
\hline $44 \mathrm{X}-1,30-32$ & $\mathrm{R}$ & M & $\mathrm{R}$ & . & & & . & . & . & R & . & $\mathrm{R}$ & . & . & $\mathrm{R}$ & . & . & . \\
\hline $44 \mathrm{X}-3,30-32$ & R & M & & . & . & . & . & . & . & . & $\mathrm{R}$ & R & . & $\mathrm{R}$ & $\mathrm{R}$ & . & . & . \\
\hline $44 \mathrm{X}-6,30-32$ & $\mathrm{R}$ & M & $\mathrm{R}$ & . & . & . & . & . & . & . & R & ? & & & $\mathbf{R}$ & . & . & . \\
\hline $44 X-7,30-32$ & R & M & R & $\mathrm{R}$ & . & . & . & . & . & $\mathbf{R}$ & ? & $\mathrm{R}$ & & $\mathrm{R}$ & : & & . & . \\
\hline $44 \mathrm{X}-\mathrm{CC}$ & R & M & $\mathrm{R}$ & . & . & . & & . & . & $\mathrm{R}$ & . & R & . & & & , & . & . \\
\hline $45 X-1,75-77$ & $\mathrm{~F}$ & M & & . & $\mathrm{R}$ & . & . & . & . & C & & $\ddot{R}$ & $\mathbf{R}$ & & . & $\mathrm{R}$ & . & . \\
\hline $45 X-3,74-76$ & $\mathrm{~F}$ & M & $\mathbf{R}$ & $\mathbf{R}$ & . & . & . & . & . & C & . & $\ddot{R}$ & R & $\mathrm{R}$ & . & $\mathrm{R}$ & . & . \\
\hline $45 X-5,74-76$ & R & M & $\mathrm{R}$ & $\mathrm{R}$ & . & . & . & . & . & $\mathrm{R}$ & . & . & & ? & . & $\ddot{R}$ & . & . \\
\hline $45 X-7,74-76$ & R & M & $\mathrm{R}$ & . & . & . & . & . & . & $\mathrm{R}$ & & . & $\mathrm{R}$ & . & . & ? & $\mathrm{R}$ & \\
\hline $45 \times-9,5-7$ & $\mathrm{R}$ & M & $\mathrm{R}$ & . & $\mathrm{R}$ & . & . & . & & $\mathrm{R}$ & . & . & & & $\mathrm{R}$ & $\mathrm{R}$ & $\mathrm{R}$ & $\mathrm{R}$ \\
\hline $45 \mathrm{X}-\mathrm{CC}$ & $\mathrm{R}$ & M & . & . & . & . & . & . & . & R & . & $\mathrm{R}$ & . & . & & . & . & \\
\hline $46 \times-1,37-39$ & $\mathrm{R}$ & M & $\mathbf{R}$ & $\mathbf{R}$ & . & . & . & . & . & $\mathrm{R}$ & . & $\mathrm{R}$ & . & . & & $\mathrm{R}$ & . & \\
\hline $46 \mathrm{X}-3,37-39$ & R & M & . & . & $\mathrm{R}$ & . & . & . & . & $\mathrm{R}$ & & R & $\mathrm{R}$ & & . & & & \\
\hline $46 \times-5,37-39$ & $\mathrm{~F}$ & M & $\mathbf{R}$ & $\mathrm{R}$ & . & . & . & . & . & $\mathrm{F}$ & . & $\mathrm{F}$ & R & . & & $\mathrm{F}$ & & \\
\hline $46 \mathrm{X}-\mathrm{CC}$ & $\mathrm{R}$ & M & R & . & . & . & . & . & & $\mathrm{R}$ & . & $\mathrm{R}$ & $\because$ & . & & : & . & \\
\hline $47 \mathrm{X}-1,28-30$ & $\mathrm{R}$ & M & . & . & . & . & . & . & & $\mathrm{R}$ & . & . & . & . & . & . & & . \\
\hline $47 \mathrm{X}-3,30-32$ & R & M & . & $\mathbf{R}$ & . & . & . & . & & & . & . & . & $\mathrm{R}$ & . & . & . & . \\
\hline $47 \mathrm{X}-5,32-34$ & R & M & . & $\mathrm{R}$ & . & . & . & . & . & & . & $\mathrm{R}$ & $\mathbf{R}$ & . & . & & $\mathrm{R}$ & R \\
\hline $47 \times-8,10-12$ & R & M & . & $\mathrm{R}$ & . & . & . & . & & . & . & $\mathrm{R}$ & $\mathbf{R}$ & . & . & . & & . \\
\hline $47 \mathrm{X}-\mathrm{CC}$ & R & $\mathrm{M}$ & . & . & . & . & . & . & . & & . & . & & . & & . & $\mathrm{R}$ & . \\
\hline $48 \mathrm{X}-\mathrm{CC}$ to $54 \mathrm{X}-\mathrm{CC}$ & Barren & & & & & & & & & & & & & & & & & \\
\hline
\end{tabular}


Table 3. Distribution of radiolarians, Hole 798C.

\begin{tabular}{|c|c|c|c|c|c|c|c|c|c|c|}
\hline $\begin{array}{l}\text { Core, } \\
\text { section, } \\
\text { interval }(\mathrm{cm})\end{array}$ & 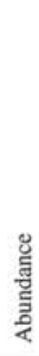 & 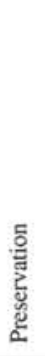 & 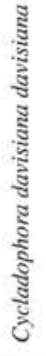 & 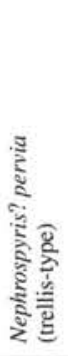 & 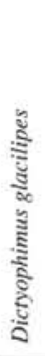 & 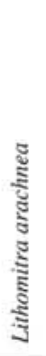 & 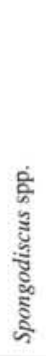 & 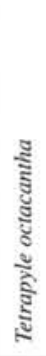 & 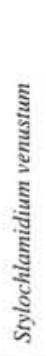 & 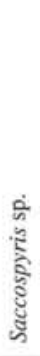 \\
\hline \multicolumn{11}{|l|}{$128-798 \mathrm{C}$ - } \\
\hline 1H-CC & $\mathrm{R}$ & M & $\mathrm{R}$ & R & . & & & & & \\
\hline $2 \mathrm{H}-\mathrm{CC}$ & R & M & . & R & . & . & . & & . & \\
\hline $3 \mathrm{H}-\mathrm{CC}$ & $\mathrm{R}$ & M & $\mathrm{R}$ & $\mathrm{R}$ & . & & . & . & . & \\
\hline 4H-CC & R & M & . & $\mathrm{R}$ & . & & . & . & . & \\
\hline SH-CC & B & . & . & . & . & & . & . & . & . \\
\hline $6 \mathrm{H}-\mathrm{CC}$ & B & : & . & & . & & & & . & \\
\hline 7H-CC & R & M & . & . & $\mathrm{R}$ & $\mathrm{R}$ & $\mathrm{R}$ & $\mathrm{R}$ & . & . \\
\hline $8 \mathrm{H}-\mathrm{CC}$ & $\mathrm{R}$ & M & . & R & $\mathrm{R}$ & . & . & & & . \\
\hline 9H-CC & B & . & . & . & . & & & & & . \\
\hline $10 \mathrm{H}-\mathrm{CC}$ & B & . & . & . & . & & . & . & . & . \\
\hline $11 \mathrm{H}-\mathrm{CC}$ & R & M & . & . & $\mathrm{R}$ & $\mathrm{R}$ & . & . & $\mathrm{R}$ & $\mathrm{R}$ \\
\hline $12 \mathrm{H}-\mathrm{CC}$ & $\mathrm{R}$ & M & . & . & $\mathrm{R}$ & & & . & . & . \\
\hline $13 \mathrm{H}-\mathrm{CC}$ & R & M & & & . & $\mathrm{R}$ & $\mathrm{R}$ & & . & . \\
\hline
\end{tabular}

tensis (Samples 128-798B-45X-1, 75-77 cm, 422.25 mbsf; and 128$799 \mathrm{~A}-46 \mathrm{X}-6,80-82 \mathrm{~cm}, 421.90 \mathrm{mbsf}$ ), which, in turn, is younger than the evolutionary transition from $S$. delmontensis to $S$. peregrina in Hole 799A (Sample 128-799A-46X-CC; 423.30 mbsf).

4. LAD of Cyrtocapsella tetrapera: The datum level of the rapid decrease of $C$. tetrapera has been widely observed from the Honshu land sections originally by Nakaseko and Sugano (1973) and subsequently by numerous workers (e.g., Takayanagi et al., 1984; Ling and Kobayashi, in press; Ling et al., 1988). The datum falls just above the base of the Denticulopsis praedimorpha (diatom) Zone at 13.3 Ma (Akiba, 1986). On the other hand, a short acme of Lithopera renzae renzae and the LAD of Eucyrtidium asanoi occur slightly below this level and are located within the upper part of the underlying Denticulopsis nicobarica (diatom) Zone (Takayanagi et al., 1984). The occurrences of radiolarians from Holes 799A and 799B is in accord with these sequences.

\section{Taxonomic Notes}

All the radiolarian taxa observed from the Japan Sea sediments have been discussed and/or adequately illustrated previously by investigators, especially from the previous DSDP cruises in the North Pacific, including Leg 31, which was the only cruise into the Sea of Japan (Ling, 1975). Therefore, in this section, all the radiolarians are listed in alphabetical order of the current nomenclature together with the original references, followed by additional references and/or remarks as necessary. Also, only biostratigraphically important forms are illustrated.

Amphirhopalum ypsilon Haeckel, 1887, p. 522; Nigrini, 1967, p. 35, pl. 31, figs. 3a-3d. (Pl. 1, Fig. 1)

Anthocorys(?) akitaensis Nakaseko. See Ling, 1971, p. 696-697, pl. 2, figs. 10-13; Ling, 1973, p. 728, pl. 8, figs. 17, 18. (Pl. 1, Fig. 2)

Remarks. Although the present taxon may be closely related to some species discussed in cycladophorid radiolarians by Lombari and Lazarus (1988), this generally known nomenclature by Japanese geoscientists is provisionally retained here until further analysis by the present author is completed.

Artostrobus annulatus (Bailey), Haeckel, 1887, p. $1481=$ Cornutella annulata Bailey, 1856, p. 3, pl. 1, figs. 5a, b.

Botryostrobus auritus-australis group, Nigrini, 1977, p. 126-128, pl. 1, figs. 2-5 (see also for the complete synonymy).
Cornutella profunda Ehrenberg, 1859, p. $31=$ C. clathrata? profund $a$ Ehrenberg, 1856, pl. 35B, fig. 21 .

Cycladophora davisiana cornutoides $($ Petrushevskaya), Ling $=$ Cycladophora davisiana var. cornutoides Petrushevskaya, 1967, p. 124-126, fig. 70, 1-3. (Pl. 1, Fig. 6)

Remarks. Petrushevskaya's variety has been raised to the subspecies of Theocalyptra davisiana by Kling (1977). However, the original figures by Petrushevskaya have a more slender, conical thoracic outline, more similar to those observed from the submarine sediments of the Bering Sea (Ling et al., 1971, pl. 2, fig. 7 only) and the Sea of Okhotsk (Ling, 1974) than those from the California coastal basins illustrated by Kling (1977, p. 217, pl. 1, fig. 20), Petrushevskaya's nomenclature is followed except it is raised to a subspecific rank.

Cycladophora davisiana davisiana Ehrenberg, Petrushevskaya, 1967, p. $122-$ 124, figs. 69, 1-7 = Cycladophora? davisiana Ehrenberg, 1862, p. 297. (PI. 1, Fig. 5)

Cyrtocapsella tetrapera Haeckel, Sanfilippo and Riedel, 1970, p. 453, pl. 1, figs. $16-18=$ Cyrtocapsa tetrapera Haeckel, 1887, p. 1512. (PI. 1, Fig. 7) Dictyophimus bicornis (Ehrenberg) Petrushevskaya, 1967, p. 72-73, fig. 41, $\mathrm{I}-\mathrm{V}=$ Lithomelissa? bicornis Ehrenberg, 186lb, p. 300; Ehrenberg, 1872b, p. 297 , pl. 2, fig. 7 . Haeckel, 1887, p. 1206.

Dictyophimus gracilipes Bailey, 1856, p. 4, pl. 1, fig. 8. See Riedel, (1958) for synonymy.

Didymocyrtis mammifera (Haeckel) Sanfilippo and Riedel, 1980, p. $1010=$ Cannartidium mammiferum Haeckel, 1887, p. 375, pl. 39, fig. 6. (Pl. 1, Fig. 8)

Didymocyrtis tetrathalamus (Haeckel) Sanfilippo and Riedel, 1980, p. 1010, text-fig. $1 \mathrm{~g}=$ Panartus tetrathalamus Haeckel, 1887, p. 378, pl. 40, fig. 3 . (PI. 1, Fig. 9)

Druppatractus acquilonius Hays, 1970, p. 214, pl. 1, figs. 4, 5. (PI. 1, Fig. 10)

Euchitonia elegans Ehrenberg, 1872a, p. 319; Ehrenberg, 1872b, p. 299, pl. 8, fig. 3 .

Euchitonia furcata Ehrenberg, 1861a, p. 767; Ling and Anikouchine, 1967, p. 1484-1486, pls. 189, 190, figs. 1-2, 5-7. (Pl. 1, Fig. 12)

Eucyrtidium asanoi Sakai, 1980, pp. 709-710, pl .7, figs. 12a, 12b-14a, 14b. (Pl. 2, Fig. 1)

Remarks. In spite of poor preservation due to diagenetic effects, the specimens can be recognized by the distinct external lumbar constriction and the two to three pores between parallel ridges in post-thoracic segments.

\section{Eucyrtidium spp.}

Remarks. Due to the rare and sporadic occurrences, except for E. asanoi, specimens belonging the genus Eucyrtidium are grouped.

Hymeniastrum euclidis Haeckel, 1887, p. 531, fig. 13.

Lithomitra arachnea (Ehrenberg), Riedel, 1958, p. 122, 123, pl. 4, figs. 7, 8. = Eucyrtidium lineatum arachneum Ehrenberg, 1862, p. 299.

Lithomitra renzae renzae Sanfilippo and Riedel, Funayama, 1988, p. 32, pl. 4, fig. 2 = Lithopera (Lithopera) renzae Sanfilippo and Riedel, 1970, p. 454, pl. 1, figs. 21-23, 27. (Pl. 2, Fig. 2)

Lychnocanoma sp. (PI. 2, Fig. 3)

Remarks. The specimens observed during the present study are identical with those reported by Funayama (1988, pl. 3, fig. 14) as L. nipponica magnacornuta Sakai (1980, p. 710, pl. 9, figs. 3, 3a) from the Noto Peninsula as having shorter thoracic legs, which is approximately the length of an apical horn. However, Sakai's specimens from the western North Pacific, judging from his holotype, possess longer and elegantly curved thoracic legs in addition to a characteristic apical horn.

Nephrospyris? pervia (Haeckel) Goll and Bjørklund, 1985, p. 125, fig. 4, D-G $=$ Tiarospyris pervia Haeckel, 1887, p. 1082, pl. 87, fig. 7 .

Remarks. Two cephalic lattice types discussed by Goll and Bjørkland (op. cit.) are also observed during the present study (PI. 1, Figs. 3 and 4). Trellis-type (figs. 4D and E): as Ceratospyris borealis Bailey, 1856, p. 3, pl. 1, fig. 3, from bottom sediments of the Sea of Kamchatka, the present-day Kamchatka Basin of the Bering Sea; as Tricera spyris? sp. Ling et al., 1971 (pl. 2, fig. 1) from the Bering Sea; Ling (1973, pl. 1, fig. 13) from the high latitude North Pacific and the Bering Sea; and Triceraspyris? sp. A from the northwestern Pacific (Ling, 1980, pl. 1, fig. 14). Perforate-type (figs. $4 \mathrm{~F}$ and G): as Triceraspyris? sp. Ling et al., 1971, pl. 2, figs. 2, 3) from the Bering Sea; Ling (1973, pl. 1, fig. 14) from 
the high latitude North Pacific and the Bering Sea; and Triceraspyris? sp. B from the northwestern Pacific (Ling, 1980, pl. 1, fig. 15).

\section{Porodiscus spp.}

Remarks. In following Haeckel's practice, both concentric and spiral ring forms are combined throughout the present study.

Saccospyris sp., see Ling et al. (1971) for synonymy and discussion. Spongaster tetras tetras Ehrenberg, 1960, p. 833; Nigrini, 1967, p. 41-43, pl. 5, figs. la, lb.

Spongaster tetras irregularis Nigrini, 1967, p. 43-44, pl. 5, fig. 2. (Pl. 2, Fig. 5)

Remarks. Although the illustrated specimens has a much sharper rectangular outline than those described originally from Indian Ocean by Nigrini (1967), or from the central North Pacific by Foreman (1975) and the western North Pacific by Morley (1985), it is considered here that they are within the morphologic range of the subspecies. All the previous records suggest that the occurrence of the present taxon is rare and biogeographic distribution limited only in middle- to high-latitude region.

\section{Spongodiscus spp.}

Remarks. Discoidal specimens with spongy cortical shell are all grouped under the present taxon except Stylochlamydium venustum.

Stichocorys delmontensis (Campbell and Clark), Sanfilippo and Riedel, 1970, p. 451, pl. 1, fig. $9=$ Eucyrtidium delmontense Campbell and Clark, 1944 , p. 56, pl. 7, figs. 19, 20. (Pl. Fig. 6)

Stichocorys peregrina (Riedel) Sanfilippo and Riedel, 1970, p. 451, pl. 1, fig. $10=$ Eucyrtidium elongatum peregrinum Riedel, 1953, p. 812-813, pl. 85, fig. 2. (PI. 2, Fig. 9)

Stylochlamydium venustum (Bailey) Haeckel, 1887, p. $515=$ Perichlamidium venustum Bailey, 1856, p. 6, figs. 16, 17.

Tetrapyle octacantha Müller, 1858, p. 33-35, pl. 2, figs. 12, 13; pl. 3, figs. 1-12. (PI. 2, Fig. 8)

Thecosphaera japonica Nakaseko, 1971, p. 61, 62, pl. 1, fig. 3a, b.

\section{ACKNOWLEDGMENTS}

The author acknowledges financial support from the National Science Foundation through Texas A\&M Research Foundation, thus enabling him to participate in the cruise and to carry out the postcruise analysis. Special thanks are due to Drs. Joyce R. Blueford and James C. Ingle, Jr., and Stanley A. Kling for kindly reviewing the manuscript, and Muhong Chen, Mohammad Fariduddin, and Lisa Paulson for technical assistance during preparation of the manuscript, the Leg 128 shipboard colleagues for stimulating discussion, and finally but not the least to the personnel of Leg 128 on the JOIDES Resolution (SEDCO/BP 471), for, without their efforts, the submarine sediments would never appear above sea level.

\section{REFERENCES}

Akiba, F., 1986. Middle Miocene to Quaternary diatom biostratigraphy in the Nankai trough and Japan trench, and modified lower Miocene through Quaternary diatom zones for middle-to-high latitudes of the North Pacific. In Kagami, H., Karig, D. E., Coulbourn, W. T., et al., Init. Repts. DSDP, 87: Washington (U.S. Govt. Printing Office), 393-481.

Bailey, J. W., 1856. Notice of microscopic forms found in the soundings of the Sea of Kamtschatka. Am. J. Sci, 22:1-6.

Berggren, W. A., Kent, D. V., and Van Couvering, J. A., 1985. The Neogene: Part 2. Neogene geochronology and chronostratigraphy. In Snelling, N. J. (Ed.), The Chronology of the Geological Record. Geol. Soc. London Mem., 10:211-260.

Campbell, A. S., and Clark, B. L., 1944. Miocene radiolarian faunas from Southern California. Spec. Pap. Geol. Soc. Am., 51:1-76.

Ehrenberg, C. G., 1854. Mikrogeologie: Das Erden und Felsen Schaffende Wirken des Unsichtbar Kleines Selbständigen Lebens auf der Erde: Leipzig (Leopold Voss).

, 1859. Kurze Characteristik der 9 neuen Genera und der 105 neuen Species des agaischen Meeres und des Tiefgrundes des Mittel-Meeres. $K$. Preuss. Akad. Wiss. Monatsber., 1958:10-41. 1861a. Über die organischen und unorganischen Mischungsverhältnisse des Meeresgrundes in 19800 Fuss Tiefe nach Lieut. Brookes Messung. K. Preuss. Akad. Wiss. Berlin Monatsber, 1860, 765-774.

, 1861b. Über die Tiefgrund-Verhältnisse des Oceans am Einegange der Davisstrasse und bei Island. K. Preuss. Akad. Wiss. Berlin Monatsber., 275-315

, 1872a. Mikrogeologische Studien als Zusammenfassung der Beobachtungen des kleinsten Lebens der Meeres-Tiefgründe aller Zonen und dessen geologischen Einfluss. K. Preuss. Akad. Wiss. Berlin Monatsber, 265-322.

, 1872b. Mikrogeologische Studien über das kleinste Leben der Meeres-Tiefgründe aller Zonen und dessen geologischen Einfluss. Abh. K. Akad. Wiss. Berlin, 131-399.

Foreman, H. P., 1975. Radiolarians from the North Pacific, Deep Sea Drilling Project, Leg 32. In Larson, R. L., Moberly, R., et al., Init. Repts. DSDP, 32: Washington (U.S. Govt. Printing Office), 579-676.

Funayama, M., 1988. Miocene radiolarian stratigraphy of the Suzu area, northeastern part of the Noto Peninsula, Japan. Contrib. Inst. Geol. Paleontol. Tohoku Univ., 91:15-41. (in Japanese)

Goll, R. M., and Bjørklund, K. R., 1985. Nephrospyris knutheieri sp. n., an extant trissocyclid radiolarian (polycystinae: Nassellarida) from the Norwegian-Greenland Sea. Sarsia, 70:103-118.

Haeckel, E., 1887. Report on the Radiolaria collected by H.M.S. Challenger during the years 1873-1876. Rep. Sci. Results Voy. H.M.S. Challenger, Zool., 18:1-1803.

Hays, J. D., 1970. Stratigraphy and evolutionary trends of Radiolaria in North Pacific deep sea sediments. In Hays, J. D. (Ed.), Geological Investigations of the North Pacific. Mem. Geol. Soc. Am., 126:185-218.

Hays, J. D., and Shackleton, N. J., 1976. Globally synchronous extinction of radiolarian Stylatractus universus. Geology, 4:649-652.

Ingle, J. C., Jr., Suyehiro, K., von Breymann, M. T., et al., 1990. Proc. ODP, Init. Repts., 128: College Station, TX (Ocean Drilling Program).

Kawai, H., 1972. Hydrography of the Kuroshio Extension. In Stommel, H., and Yoshida, K. (Eds.), Kuroshio: Physical Aspects of the Japan Current: Seattle (Univ. Washington Press), 235-353

Kling, S. A., 1977. Local and regional imprints on radiolarian assemblages from California coastal basin sediments. Mar. Micropaleont., 2:207-221.

Koizumi, I., 1975. Neogene diatoms from the western margin of the Pacific Ocean, Leg 31, Deep Sea Drilling Project. In Karig, D. E., Ingle, J. C., Jr., et al., Init. Repts. DSDP, 31: Washington (U.S. Govt. Printing Office), 779-819.

Koizumi, I., and Tanimura, Y., 1985. Neogene diatom biostratigraphy of the middle latitude western North Pacific, Deep Sea Drilling Project Leg 86. In Heath, G. R., Burckle, L. H., et al., Init. Repts. DSDP, 86: Washington (U.S. Govt. Printing Office), 269-300.

Ling, H. Y., 1971. Silicoilagellates and ebridians from the Shinzan diatomaceous mudstone member of the Onnagawa Formation (Miocene), Northeast Japan. In Farinacci, A. (Ed.), Proc. 2nd Planktonic Conf. Roma. Rome (Ed. Technosci.), 689-703.

1973. Radiolaria: Leg 19 of the Deep Sea Drilling Project. In Creager, J. S., Scholl, D. W., et al., Init. Repts. DSDP, 19: Washington (U.S Govt. Printing Office), 777-797.

1974. Polycystine Radiolarian and silicoflagellates from surface sediments of the Sea of Okhotsk. Bull. Geol. Surv. Taiwan, 24:1-11.

, 1975. Radiolaria: Leg 31 of the Deep Sea Drilling Project. In Karig, D. E., Ingle, J. C., Jr., et al., Init. Repts. DSDP, 31: Washington (U.S. Govt. Printing Office), 703-761.

, 1980. Radiolarians from the Emperor Seamounts of the Northwest Pacific, Leg 55 of the Deep Sea Drilling Project. In Jackson, E. D., Koizumi, I., et al., Init. Repts. DSDP, 55: Washington (U.S. Govt. Printing Office), 365-373.

Ling, H. Y., and Anikouchine, W. A., 1967. Some spumellarian Radiolaria from the Java, Philippine, and Mariana Trenches. J. Paleontol., 41:1481-1491.

Ling, H. Y., Ingle, J. C., Jr., and Kim, B. K., 1988. Miocene siliceous biostratigraphy and magnetostratigraphy from the Pohang area, Korea. J. Paleontol. Soc. Korea, 4:1-5.

Ling, H.Y., and Kobayashi, H., in press. Geological significance of siliceous microfossils from Dogo, Oki Islands. In Ishizaki, K., and Saito, T. (Eds.), Centenary of Japanese Micropaleontology: Tokyo (Terra Sci. Publ.).

Ling, H. Y., Stadum, C. J., and Welch, M. L., 1971. Polycystine Radiolaria from Bering Sea surface sediments. In Farinacci, A. (Ed.), Proc. 2nd Planktonic Conf., Roma. Rome (Ed. Technosci.), 705-729. 
Lombari, G., and Lazarus, D. B., 1988. Neogene cycladophorid radiolarians from North Atlantic, Antarctic, and North Pacific deep-sea sediments. Micropaleontology, 34:97-135.

Morley, J. J., 1985. Radiolarians from the Northwest Pacific, Deep Sea Drilling Project Leg 86. In Heath, G. R., Burckle, L. H., et al., Init. Repts. DSDP, 86: Washington (U. S. Govt. Printing Office), 399-422.

Müller, J., 1858. über die Thalassicollen, Polycystinen und Acanthometren des Mittelmeeres. K. Preuss. Akad. Wiss. Berlin, Abh., 1-62.

Nakaseko, K., 1971. On some species of the genus Thecosphaera from the Neogene Formation, Japan. Sci. Repts., Osaka Univ. Coll. Gen. Educ., 20:59-66.

Nakaseko, K., and Sugano, K., 1973. Neogene radiolarian zonation in Japan. Chishitsugaku Ronshu, 8:23-33.

Nigrini, C., 1967. Radiolaria in pelagic sediments from the Indian and Atlantic Oceans. Bull. Scripps Inst. Oceanogr., 11:1-125.

- 1977. Tropical Cenozoic Artostrobiidae (Radiolaria). Micropaleontology, 23:241-269.

Petrushevskaya, M. G., 1967. Antarctic spumelline and nasselline radiolarians (in collections of the Soviet Antarctic Expedition). Explorations of the Fauna of the Sea, Zool. Inst. Akad. Nauk, USSR, 4:5-186.

Riedel, W. R., 1953. Mesozoic and late Tertiary Radiolaria of Rotti. $J$. Paleontol., 27:805-813.

, 1958. Radiolaria in Antarctic sediments. Rep. B.A.N.Z. Antarct. Res. Exped., Ser. B, 6:217-255.

Riedel, W. R., and Sanfilippo, A., 1978. Stratigraphy and evolution of tropical Cenozoic radiolarians. Micropaleontology, 24:61-96.

Sakai, T., 1980. Radiolarians from sites 434,435, and 436, Northwest Pacific Leg 56, Deep Sea Drilling Project. In von Huene, R., Nasu, N., et al., Init.
Repts. DSDP, 56, 57 (Pt. 2): Washington (U.S. Govt. Printing Office), 695-733.

Sanfilippo, A., and Riedel, W. R., 1970. Post-Eocene "closed" theoperid radiolarians. Micropaleontology, 16:446-462.

1980. A revised generic and suprageneric classification of the Artiscins (Radiolaria). J. Paleontol., 54:1008-1011.

Sanfilippo, A., Westberg-Smith, M. J., and Riedel, W. R., 1985. Cenozoic radiolaria. In Bolli, H. M., Saunders, J. B., and Perch-Nielsen, K. (Eds.), Plankton Stratigraphy: Cambridge (Cambridge Univ. Press), 631-712.

Takayanagi, Y., Oda, M., Hasegawa, S., Honda, N., Maruyama, T., and Funayama, M., 1984. Some Middle Miocene planktonic microfossil datum planes in northern Honshu, Japan: their paleoceanographic implications. Palaeogeogr., Palaeoclimatol., Palaeoecol., 46:71-84.

Takayanagi, Y., Sakai, T., Qda, M., Hasegawa, S., and Tanimura, Y., 1979. Problems in microbiostratigraphy for geohistorical inquiry on the Sea of Japan. Nihoukai, 10:91-102 (in Japanese).

Tamaki, K., and Pisciotto, K., Allan, J., et al., 1990. Proc. ODP Init. Repts. 127: College Station, TX (Ocean Drilling Program).

Theyer, F., Mato, C. Y., and Hammond, S. R., 1978. Paleomagnetic and geochronologic calibration of latest Oligocene to Pliocene radiolarian events, equatorial Pacific. Mar. Micropaleontol., 3:377-395.

Date of initial receipt: 22 May 1991

Date of acceptance: 16 August 1991

Ms 127/128B-125 
Table 4. Distribution of radiolarians, Hole 799A.

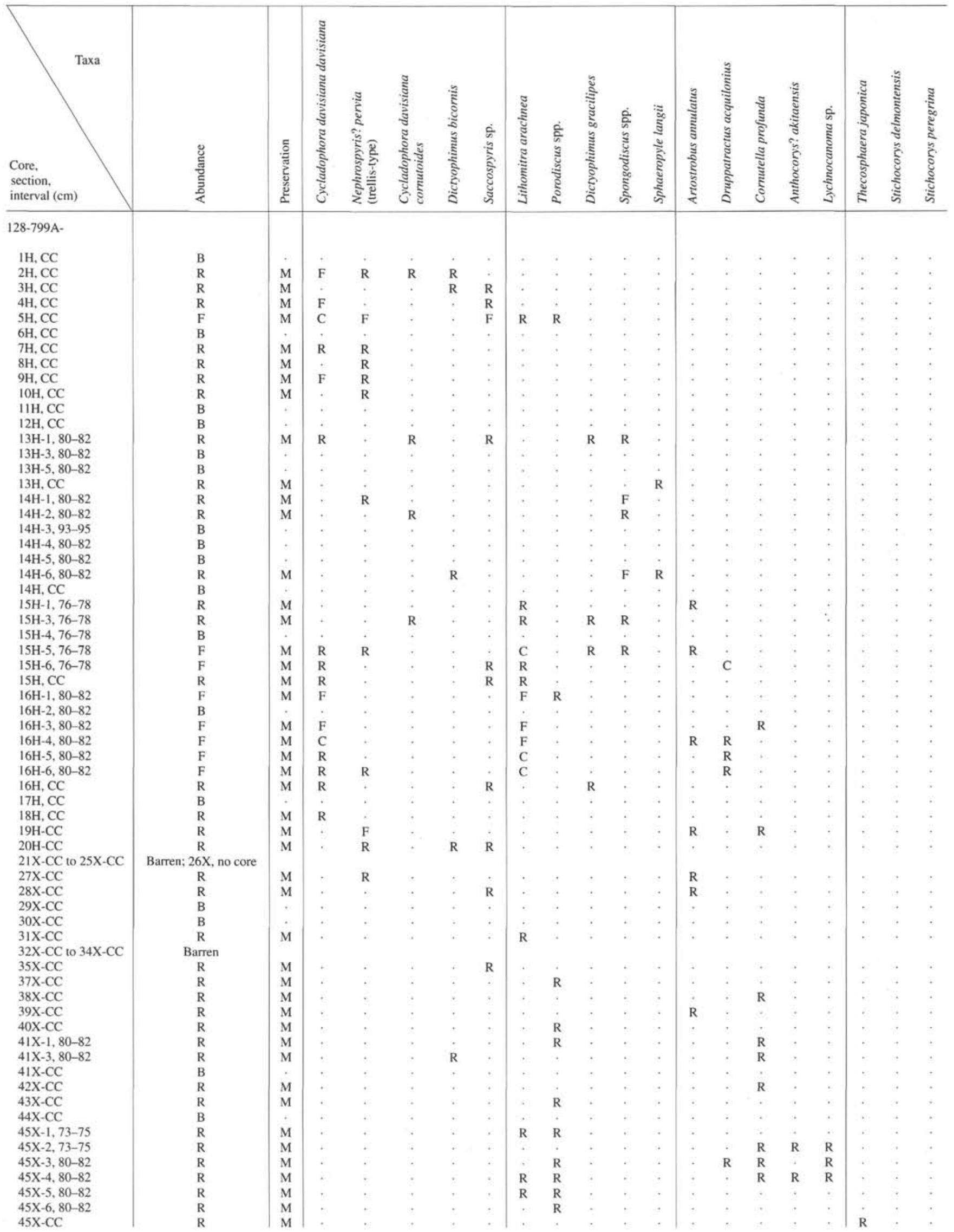


Table 4 (continued).

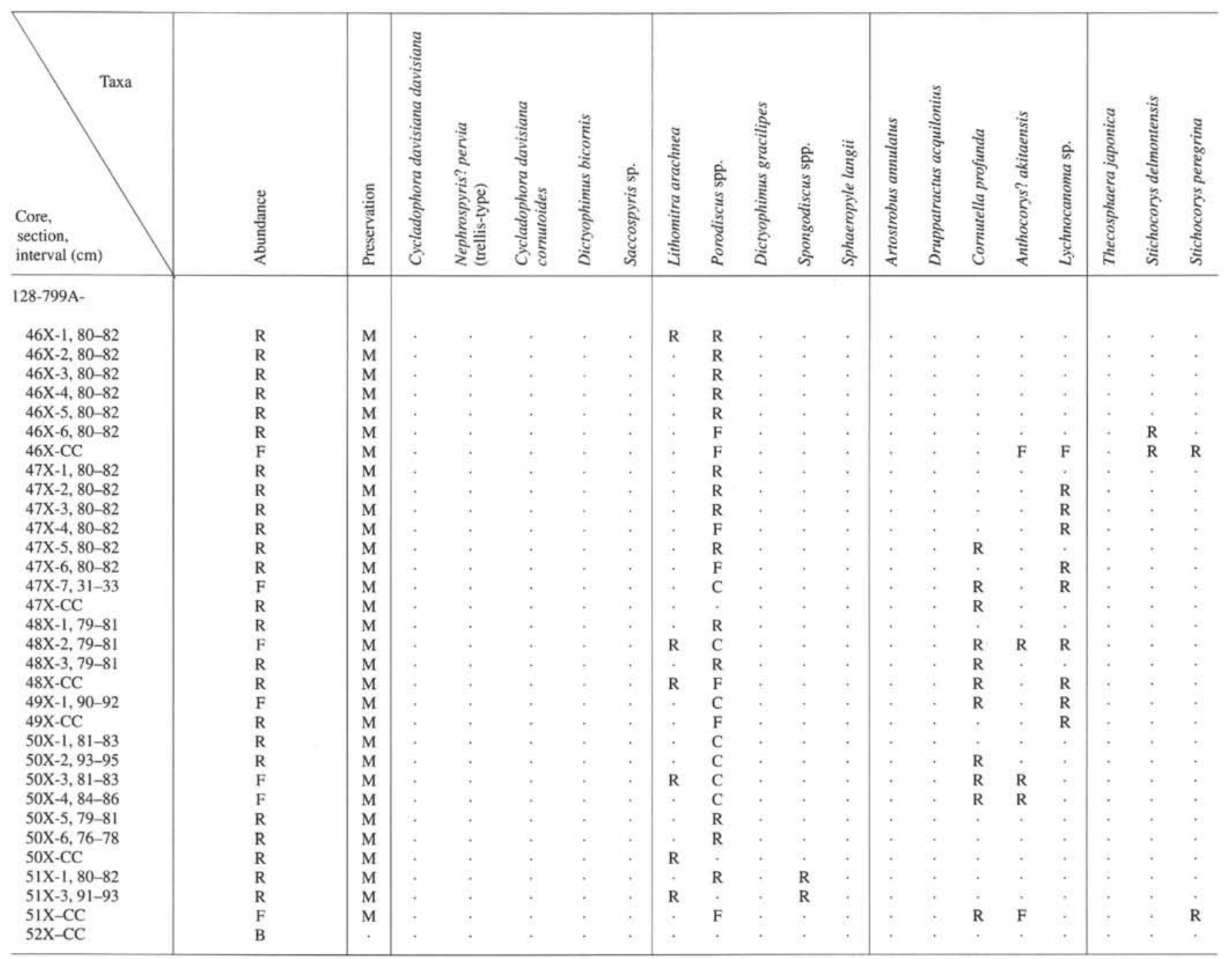

Table 5. Distribution of radiolarians, Hole 799B.

\begin{tabular}{|c|c|c|c|c|c|c|c|c|c|}
\hline $\begin{array}{l}\text { Core, } \\
\text { section, } \\
\text { interval }(\mathrm{cm})\end{array}$ & $\begin{array}{l}\text { ¿ } \\
\text { 总 } \\
\text { है }\end{array}$ & 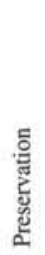 & 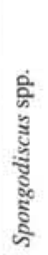 & 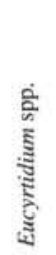 & 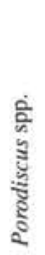 & 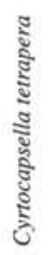 & 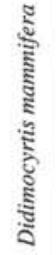 & 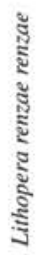 & 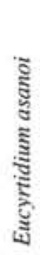 \\
\hline \multicolumn{10}{|l|}{ 128-799B- } \\
\hline SR-CC & R & $P$ & $R$ & . & . & . & & . & . \\
\hline $6 \mathrm{R}-\mathrm{CC}$ & B & . & . & . & . & . & & & \\
\hline $7 \mathrm{R}-\mathrm{CC}$ & $\mathrm{R}$ & P & $\mathrm{R}$ & R & . & . & . & . & \\
\hline 8R-CC & B & . & & . & . & & . & . & . \\
\hline 9R-CC & $\mathrm{R}$ & P & & . & $\mathrm{R}$ & & . & . & \\
\hline 10R-CC & $\mathrm{R}$ & P & & . & $\mathrm{R}$ & & . & & . \\
\hline 3IR-CC & $\mathrm{R}$ & P & . & . & . & $\mathrm{R}$ & . & . & \\
\hline $32 \mathrm{R}-\mathrm{CC}$ & $\mathrm{R}$ & P & . & . & . & $\mathrm{R}$ & . & . & . \\
\hline 38R-CC & $\mathrm{R}$ & P & . & . & . & $\mathrm{R}$ & & . & \\
\hline $48 \mathrm{R}-\mathrm{CC}$ & F & P & . & $\mathrm{R}$ & . & $\mathrm{F}$ & $\mathrm{R}$ & $\mathrm{R}$ & $\mathrm{R}$ \\
\hline 50R-CC & $\mathrm{R}$ & P & $\mathrm{R}$ & . & . & . & $\cdot$ & . & . \\
\hline
\end{tabular}




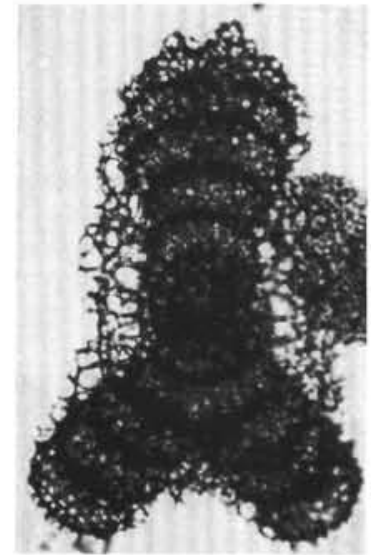

1
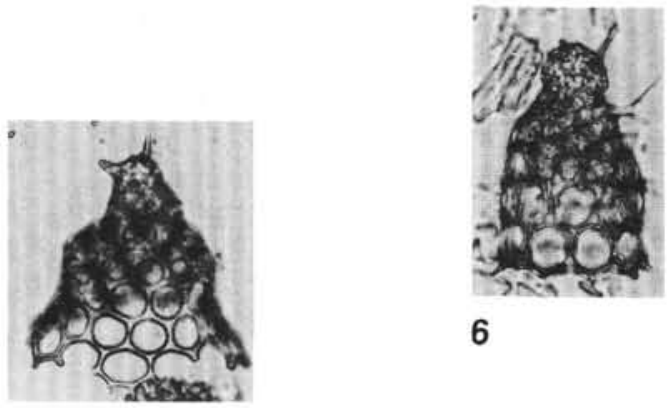

6

5

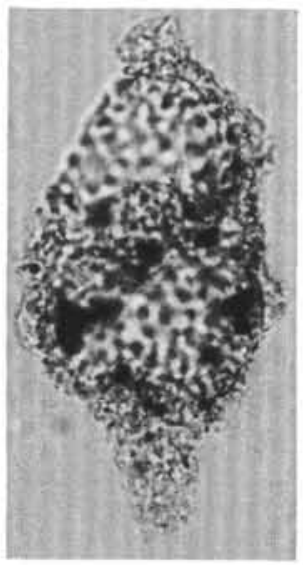

8

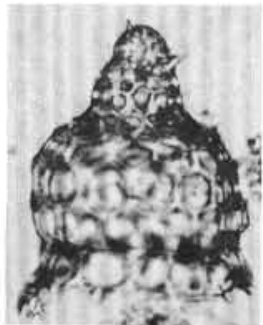

2

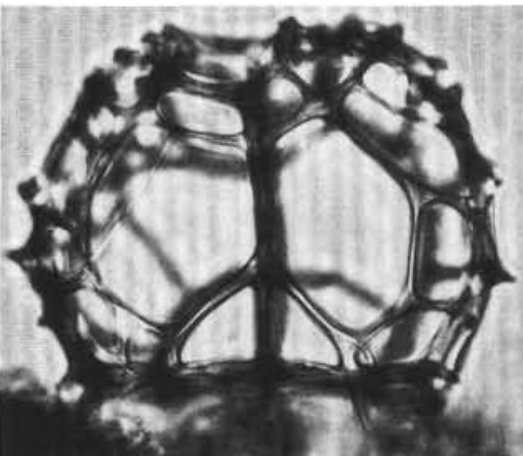

3

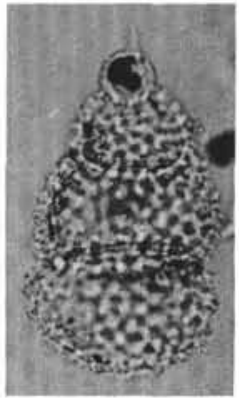

7

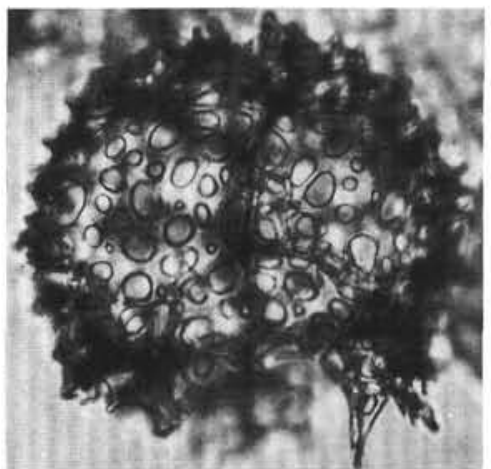

4

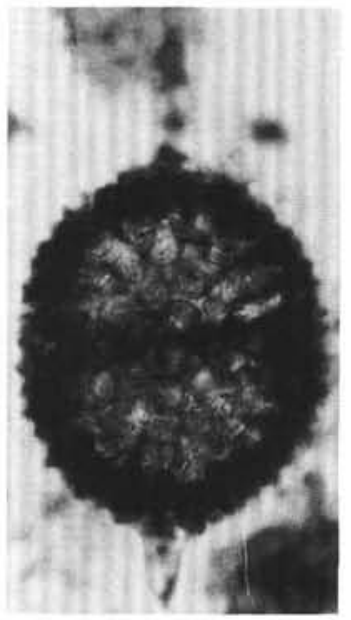

10

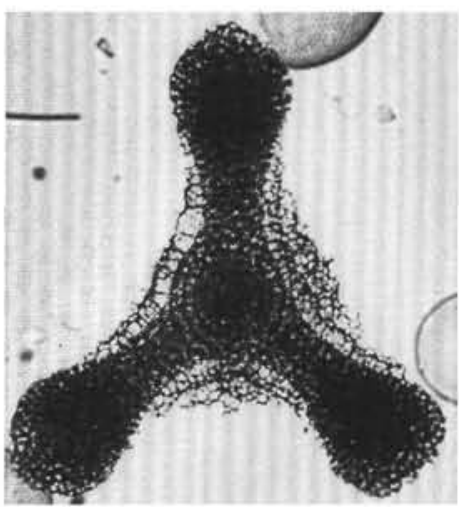

11

Plate 1. Magnification 200x unless otherwise indicated 1. Amphirhopalum ypsilon, Sample 128-798A-5H-3, 29-31 cm, R-1 (V17/3), 160×. 2. Anthocorys(?) akitaensis Sample 128-799A-51R-CC, R-1 (G29/1). 3. Nephrospyris? pervis (trellis-type), Sample 128-798A-1H-1, 30-32 cm, R-1 (R18/0), 340X. 4. Nephrospyris? pervis, (perforate-type), Sample 128-798A-13H-3,31-33 cm, R-1 (Y28/1), 340x. 5. Cycladophora davisiana, Sample 128-799A-15H-5, 765-78 cm, R-1 (S40/1). 6. C. d. cornutoides, Sample 128-799A-14H-2, 80-82 cm, R-1 (V38/4), 250x. 7. Cyrtocapsella tetrapera, Sample 128-799B-48R-CC, R-2 (R16/4). 8. Didymocyrtis mammifera, Sample 128-799B-48R-CC, R-1 (Q39/0), 300x. 9. D. tetrathalamus, Sample 128-798A-3H-3, 28-30 cm, R-1 (F23/1), 160X. 10. Druppatractus acquilonis, Sample 128-799A-15H-6, 76-78 cm, R-1 (O16/2) 11. Euchitonia furcata, Sample 128-798A-2H-1, 29-31 cm, R-1 (F21/1), 140X . 


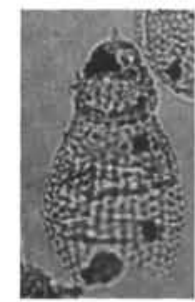

1

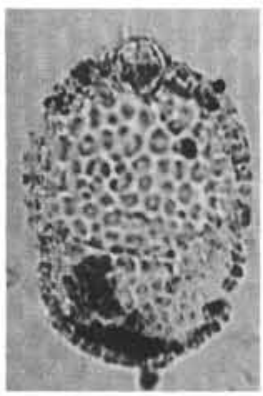

2

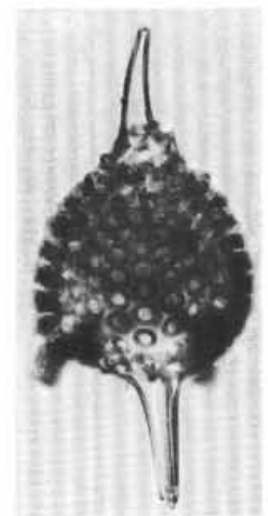

3

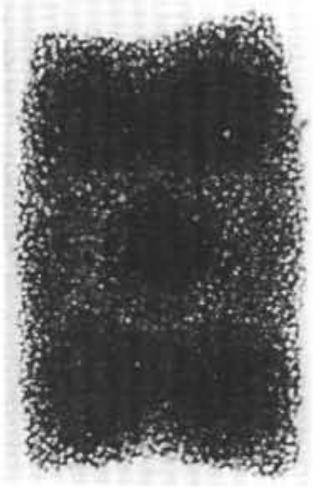

5

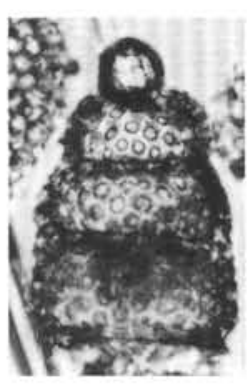

6

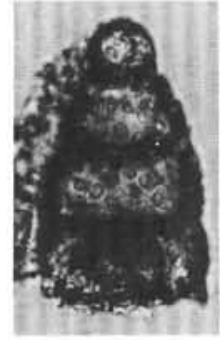

7

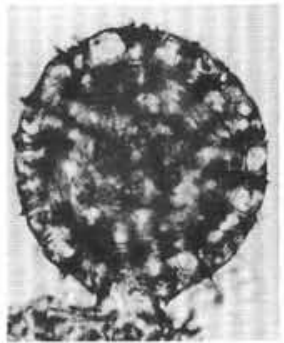

4

Plate 2. Magnification $200 \times$ unless otherwise indicated. 1. Eucyrtidium asanoi, Sample 128-799B-48R-CC, R-2 (O21/1). 2. Lithopera renzae renzae, Sample 128-799B-48R-CC, R-1 (U22/1). 3. Lychnocanoma sp., Sample 128-799A-47R-3, 80-82 cm, R-1 (G7/0). 4. Sphaeropyle langii, Sample 128799A-13H-CC, R-1 (X33/3), 250X . 5. Spongaster tetras irregularis, Sample 128-798A-2H-1, 29-31 cm, R-1 (G16/4) 160X. 6. Stichocorys delmontensis, Sample 128-799A-46X-CC, R-3 (O33/1), 250×. 7. Stichocorys peregrina, Sample 128-799A-46X-CC, R-2 (M33/3), 250x. 8. Tetrapyle octacantha, Sample 128-798A-2H-1, 29-31 cm, R-1 (D38/4). 